\title{
Regional lung metabolic profile in a piglet model of cardiopulmonary bypass with circulatory arrest
}

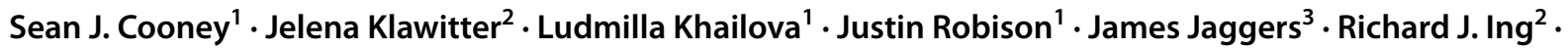 \\ Scott Lawson ${ }^{4}$. Benjamin S. Frank ${ }^{1}$. Suzanne Osorio Lujan ${ }^{1} \cdot$ Jesse A. Davidson ${ }^{1,5}$
}

Received: 31 May 2021 / Accepted: 14 September 2021 / Published online: 22 September 2021

(c) The Author(s), under exclusive licence to Springer Science+Business Media, LLC, part of Springer Nature 2021

\begin{abstract}
Introduction Acute lung injury is common following cardiopulmonary bypass and deep hypothermic circulatory arrest for congenital heart surgery with the most severe injury in the dorsocaudal lung. Metabolomics offers promise in deducing mechanisms of disease states, providing risk stratification, and understanding therapeutic responses in regards to CPB/ DHCA related organ injury.

Objectives Using an infant porcine model, we sought to determine the individual and additive effects of CPB/DHCA and lung region on the metabolic fingerprint, metabolic pathways, and individual metabolites in lung tissue.

Methods Twenty-seven infant piglets were divided into two groups: mechanical ventilation + CPB/DHCA $(n=20)$ and mechanical ventilation only $(n=7)$. Lung tissue was obtained from dorsocaudal and ventral regions. Targeted analysis of 235 metabolites was performed using HPLC/MS-MS. Data was analyzed using Principal Component Analysis (PCA), Partial Least Square Discriminant Analysis (PLS-DA), ANOVA, and pathway analysis.

Results Profound metabolic differences were found in dorsocaudal compared to ventral lung zones by PCA and PLS-DA ( $\mathrm{R} 2=0.7 ; \mathrm{Q} 2=0.59 ; p<0.0005)$. While overshadowed by the regional differences, some differences by exposure to CPB/ DHCA were seen as well. Seventy-four metabolites differed among groups and pathway analysis revealed 20 differential metabolic pathways.

Conclusion Our results demonstrate significant metabolic disturbances between dorsocaudal and ventral lung regions during supine mechanical ventilation with or without $\mathrm{CPB} / \mathrm{DHCA}$. CPB/DHCA also leads to metabolic differences and may have additive effects to the regional disturbances. Most pathways driving this pathology are involved in energy metabolism and the metabolism of amino acids, carbohydrates, and reduction-oxidation pathways.
\end{abstract}

Keywords Acute lung injury $\cdot$ Cardiopulmonary bypass $\cdot$ Kynurenine metabolism $\cdot$ Congenital heart disease surgery · Metabolomics · Pathway analysis

\section{Introduction}

Jesse A. Davidson

jesse.davidson@childrenscolorado.org

1 Department of Pediatrics, University of Colorado School of Medicine, Aurora, CO, USA

2 Department of Anesthesiology, University of Colorado, Aurora, CO, USA

3 Department of Surgery, University of Colorado, Aurora, CO, USA

4 Heart Institute, Children's Hospital Colorado, Aurora, CO, USA

5 Children's Hospital Colorado, 13123 East 16th Avenue, Box 100, Aurora, CO 80045, USA
Approximately $1 \%$ of children born in the United States and Europe are diagnosed with congenital heart disease (Benjamin et al., 2018). Of those, $25 \%$ have critical congenital heart disease that requires surgery or catheter intervention within the first year of life (Benjamin et al., 2018). Unfortunately, the morbidity rate for these surgeries remains high with frequent complications leading to prolonged hospital length of stay (Pasquali et al., 2014). For many of these surgeries, cardiopulmonary bypass (CPB) is used to maintain perfusion and deep hypothermic circulatory arrest (DHCA) or selective cerebral perfusion may be used if a bloodless field is required. However, CPB and DHCA directly 
contribute to postoperative inflammation and multiorgan injury (Kozik \& Tweddell, 2006).

Acute lung injury (ALI) is among the lesser understood complications of CPB/DHCA but is an important determinant of morbidity and mortality (Apostolakis et al., 2010). Post-CPB ALI occurs in over 35\% of cases (Liu et al., 2012; Shi et al., 2014) and can lead to prolonged mechanical ventilation and hospital stays (Shi et al., 2008). In severe cases, the mortality rate is approaches $33 \%$ (Khemani et al., 2019). The lungs, particularly those of neonates and infants (Kozik \& Tweddell, 2006), are uniquely vulnerable to injury during CPB. The pulmonary vasculature has substantially diminished circulation with only bronchial artery supply during $\mathrm{CPB}$, ventilation is often stopped or decreased during $\mathrm{CPB}$, the lungs filter the venous circulation thus receiving all inflammatory products circulating from CPB, lung capillaries are small and trap aggregates of activating substances and cells in the lung, and large pools of neutrophils reside in the lungs that are immediately present to contribute to injury (Apostolakis et al., 2010; Kozik \& Tweddell, 2006). This constellation leads to increased pulmonary vascular resistance, reduced surfactant activity, atelectasis, and pulmonary edema (Apostolakis et al., 2010; Kozik \& Tweddell, 2006; Stephens et al., 2013). Furthermore, supine positioning leads to regional differences in lung ventilation and perfusion, which results in differences in injury patterns in the dorsal regions compared to ventral regions (Altemeier et al., 1985; Carvalho et al., 1985; Freitas et al., 2016). This heterogeneous injury contributes to overall lung inflammation, hypoxemia, and intrapulmonary shunting (Tojo et al., 2015). Particularly, little is known on the early metabolic changes induced by mechanical ventilation or $\mathrm{CPB} / \mathrm{DHCA}$ nor if these changes may be localized regionally.

Metabolomics is an emerging field that examines changes in patterns of metabolite production to deduce mechanisms of disease states, provide risk stratification, and understand therapeutic responses (Johnson et al., 2016). Metabolites are small molecules that are the end products of metabolism and reflect protein activity (Mussap et al., 2013). They drive the essential functions of the cell including signaling, energy production and storage, and reduction-oxygenation reactions (Mussap et al., 2013). Patterns of metabolite expression are related to activity of specific biochemical pathways (Bowler et al., 2017; Johnson et al., 2016). In the study of the lung metabolome, indirect samples of lung metabolic activity such as urine, blood, exhaled breath particulate tissues, and bronchoalveolar lavage fluid (BALF) are most often used (Bowler et al., 2017); however, the interpretation of metabolite levels in these samples is less clear than direct samples of lung tissue (Johnson et al., 2016). There are far fewer studies that use lung tissue for metabolomic profiling.

In this study, we used a porcine model of early infant $\mathrm{CPB} / \mathrm{DHCA}$ and performed targeted metabolomic profiling on lung tissue to compare key metabolic changes induced by (1) CPB/DHCA versus mechanical ventilation only controls (MV) and (2) dorsocaudal versus ventral lung regions. We hypothesized that CPB/DHCA would induce broad metabolic changes in the lung compared to the MV group and that the dorsocaudal region of the lung would demonstrate unique metabolic disturbances compared to the ventral region of the lung. We also sought to identify key metabolic pathways and individual metabolites that drove differences between these groups.

\section{Materials and methods}

Please refer to the online supplement for full details.

\subsection{CPB/DHCA piglet model}

The animal protocol used was approved by the Institutional Animal Care and Use Committee of the University of Colorado (Protocol Number: 107715(02)1D) in accordance with the Guide for the Care and Use of Laboratory Animals and the ARRIVE guidelines. Twenty-seven infant female piglets $(5-10 \mathrm{~kg})$ were included in an animal protocol. Water was supplied ad libitum and the feeding (Teklad 8753, Envigo, Madison, WI, U.S.A.) schedule was based on an activity factor of 1.2, for slight growth/gain and maintenance, $100 \mathrm{~g}$ feed twice daily (200 g/day).

The animals were divided into two groups, CPB/DHCA $(n=20)$ and MV controls $(n=7)$. The surgical methods have previously been published (Davidson et al., 2019). Briefly, peripheral CPB was achieved under isoflurane anesthesia. During DHCA, the animals were cooled to $22{ }^{\circ} \mathrm{C}$ for $75 \mathrm{~min}$. After rewarming and CPB separation, the piglets were provided ICU care for $4 \mathrm{~h}$ then euthanized. For the MV group, animals were invasively mechanically ventilated under anesthesia with the same ventilator parameters and medications for anesthesia and euthanasia for $7 \mathrm{~h}$ without undergoing CPB/DHCA. Groups were then subdivided based on location of lung tissue biopsy to yield 4 sets of samples: ventral $\mathrm{CPB} / \mathrm{DHCA}$, dorsocaudal CPB/DHCA, ventral MV, and dorsocaudal MV. In our piglets in a supine position, the ventral lung region is considered gravity independent and the dorsal lung region is considered gravity dependent.

\subsection{Histology}

Right upper and lower lobe lung tissue was collected from each animal and fixed overnight in $10 \%$ formalin, paraffinembedded, and sectioned at $4 \mu \mathrm{m}$. Serial sections were stained with hematoxylin-eosin (H\&E) and evaluated for severity of lung injury by a blinded pathologist per the ATS recommendations (Matute-Bello et al., 2011). 


\subsection{Wet/dry ratio}

Separate tissue samples $(2 \times 2 \mathrm{~cm})$ from the right upper and lower portions of the lungs were weighed and left to dry for 5-7 days at $65^{\circ} \mathrm{C}$ until constant weight was achieved to measure wet/dry ratios.

\subsection{RNA preparation, $\mathrm{RT}$, and real-time PCR}

Total RNA was isolated from lung tissue (right upper and lower lobe tissue samples snap frozen in liquid $\mathrm{N}_{2}$ ) using the RNeasy Mini Kit (Qiagen, Santa Clarita, CA) as described in the manufacturer's protocol. RNA concentrations were quantified at $260 \mathrm{~nm}$, and the purity and integrity were determined using a NanoDrop. RT and real-time PCR assays were performed to quantify steady-state mRNA levels of IL-6. cDNA was synthesized from $0.5 \mu \mathrm{g}$ of total RNA. Predeveloped TaqMan primers and probes (Applied Biosystems, Foster City, CA) were used for detection. Reporter dye emission was detected by an automated sequence detector combined with ABI Prism 7300 Real Time PCR System (Applied Biosystems, Foster City, CA). Real-time PCR quantification was performed with TaqMan b-actin controls and relative mRNA expression calculated using the $2^{-\Delta \Delta \mathrm{CT}}$ method.

\subsection{Tissue harvest and sample preparation}

Collected lung tissue samples were extracted according to a published protocol (Davidson et al., 2018). Briefly, frozen samples were ground with tissue grinder in $500 \mu \mathrm{L}$ ice-cold methanol, sonicated for $10 \mathrm{~min}$ and centrifuged at $14,000 \mathrm{~g}$ for $10 \mathrm{~min}$ at $4{ }^{\circ} \mathrm{C}$. The precipitant was reextracted with additional $500 \mu \mathrm{L}$ ice-cold methanol, the supernatants were combined, centrifuged, and dried in a SpeedVac concentrator (Savant, ThermoFisher, Waltham, MA). Samples were reconstituted with $60 \mu \mathrm{L}$ water/methanol (80:20, volume/ volume).

\subsection{Mass spectrometry}

Multiple reaction monitoring (MRM) of 184 metabolites using a positive/negative ion-switching high-performance liquid chromatography-tandem mass spectrometry (5500 QTRAP HPLC-MS/MS (Yuan et al., 2012)) was used for analysis. Yuan et al. have provided reproducibility and extraction recovery testing in FFPE-embedded normal lung and kidney tissue as well as in kidneys from acute myeloid leukemia patients and lung tissues from patients with lymphangioleiomyomatosis lung disease. Our samples were immediately frozen and processed quickly using the published protocol thus no negative impact regarding metabolites stability or recovery should be expected.

\subsection{Spectra analysis}

MultiQuant (v2.1.1., Sciex, Foster City, CA) software was used for data processing of 235 unique metabolites in plasma. For between-sample normalization, the intensity values for each sample were summed, and the median value of the sums across all samples were determined. Tune and quality control samples were evenly distributed during the batches. The intensity values of each sample were then scaled such that the sum of the scaled intensities equaled the median value of all samples. Normalized intensity values were then $\log _{2}$ transformed to reduce the influence of extreme values and to meet the homogeneity of variance assumption.

\subsection{Statistics}

Relative peak intensities were analyzed using MetaboAnalyst 4.0 (http://www.metaboanalyst.ca), which is run on the statistical package R (v3.4.3) (Chong et al., 2019). Where applicable, metabolites are named and grouped as listed on the Human Metabolome Database (HMDB) (http://www. hmdb.ca) (Wishart et al., 2018). Principle component analysis (PCA) was performed first to see how data clustered in an unsupervised analysis. Partial least-squares discriminant analysis (PLS-DA) was then used to identify the features that discriminated between the preassigned groups. Variable importance of projection (VIP) was used to identify the most influential variables to the discrimination in PLS-DA. A one-way analysis of variance (ANOVA) was performed to determine which metabolites differed significantly among groups. P-value was adjusted for false discovery rate (FDR) of 0.05. Fisher's Least Significant Difference (LSD) posthoc test was performed to determine if the relative peak intensity was significantly elevated by region (dorsocaudal region versus ventral region), CPB/DHCA versus $\mathrm{MV}$, or additive. Metabolites with an additive effect demonstrated effects of both CPB/DHCA and lung region in at least one group. Pathway analysis was performed using GlobalANCOVA (Hummel et al., 2007) for Metabolite Set Enrichment Analysis (MSEA) and Relative-Betweeneness centrality for topographic analysis. SMPDB (https://www.smpdb.ca) (Frolkis et al., 2010; Jewison et al., 2014) was used for the pathway library. Descriptions from this database were used to categorize pathways based on common features. Cut-off for statistical significance for pathway analysis were a HolmBonferonni adjusted $\mathrm{p}$-value $<0.000556$ and an impact factor $\geq 0.1$. All artwork was created with MetaboAnalyst 4.0 with labels and size edited with Apple Preview version 11.0. 


\section{Results}

\subsection{Samples and initial metabolite data preparation}

There were 20 animals who underwent $\mathrm{CPB} / \mathrm{DHCA}$ and 7 animals who underwent MV. After the samples from the ventral and dorsocaudal regions were obtained, the final samples included the following: (1) in the MV group, 6 samples from the ventral region and 7 samples from the dorsocaudal region and (2) in the CPB/DHCA group, 18 samples from the ventral region and 20 samples from the dorsocaudal region.

On gross inspection, the lungs from all animals in both the CPB/DHCA and MV control groups showed significant discoloration in the dorsocaudal region (see Supplemental Fig. S1). Histologic examination of the dorsocaudal lung compared to the ventral lung demonstrated a marked difference in atelectasis as well as increased septal thickening and the scattered presence of intra-alveolar inflammatory cells in both the CPB/DHCA animals and MV controls. (Supplemental Fig. S2). The dorsocaudal region demonstrated a higher wet-to-dry ratio compared to the ventral lung within both the MV (median 4.9 [IQR 4.7, 5] versus 4.2 [IQR 3.8, 4.4 ]; $p=0.02$ ) and CPB/DHCA group (median 4.4 [IQR 3.9,

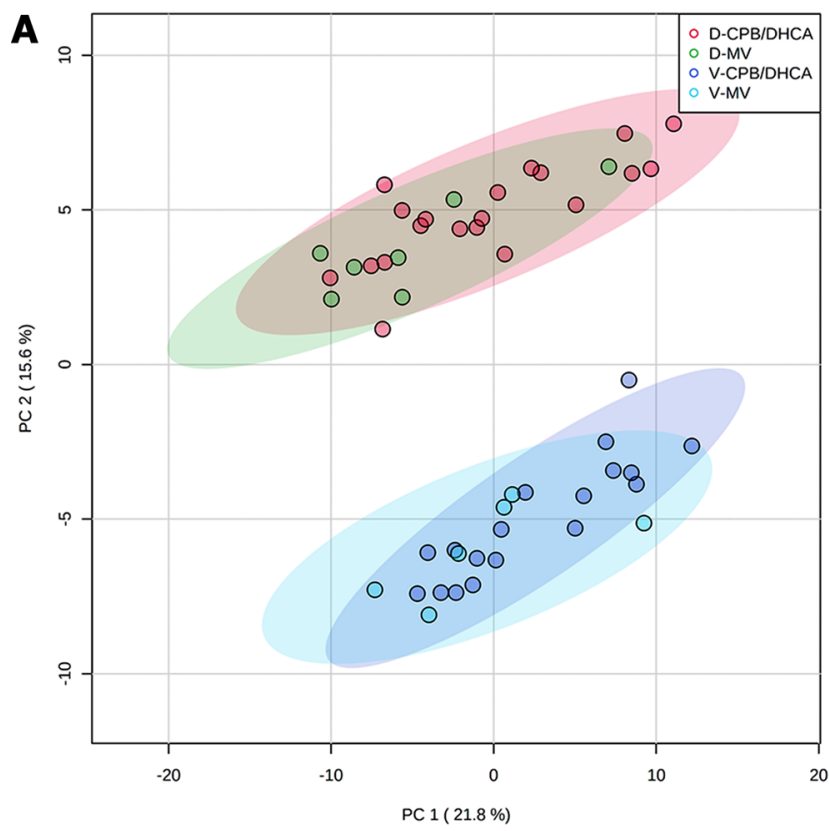

Fig. 1 Principal component analysis score plot and 2-dimensional partial least squares discriminant analysis score plot. A Principal Component Analysis Score Plot. Principal components 1 and 2 are shown. B 2-Dimensional Partial Least Squares Discriminant Analysis Score Plot. Components 1 and 2 are shown. Circles reflect samples for each group as described in the legend. Shaded regions represent
4.8 ] versus 3.5 [IQR 3.2, 3.8]; $p<0.0001)$. There was no significant difference in wet-to-dry ratio between the CPB/ DHCA and MV groups. Collectively these findings suggest a different microenvironment in the dorsocaudal region and support the concept of regional metabolomic analysis. Additionally, IL-6 mRNA expression was significantly elevated in the dorsocaudal lung of $\mathrm{CPB} / \mathrm{DHCA}$ animals compared to MV controls (17-fold increase; $p=0.01$ ) but not in the ventral lung, demonstrating the potential for synergistic effects between lung region and exposure to CPB/DHCA that should also be accounted for during metabolomic analysis.

Two hundred and thirty-five metabolites were initially targeted. After removing unstable metabolites or metabolites that were below the limits of quantification for the large majority of samples, 175 metabolites remained.

\subsection{Unsupervised and supervised metabolomic fingerprinting}

To begin the analysis, unsupervised PCA was performed to see how the data segregated independent of sample group identification. The analysis revealed clear differentiation between regions of the lung based on the metabolic fingerprint. As shown in Fig. 1A, the data differentiated along both PC1 (21.8\%) and PC2 (15.6\%) demonstrating distinct metabolic patterns between the ventral and dorsocaudal regions

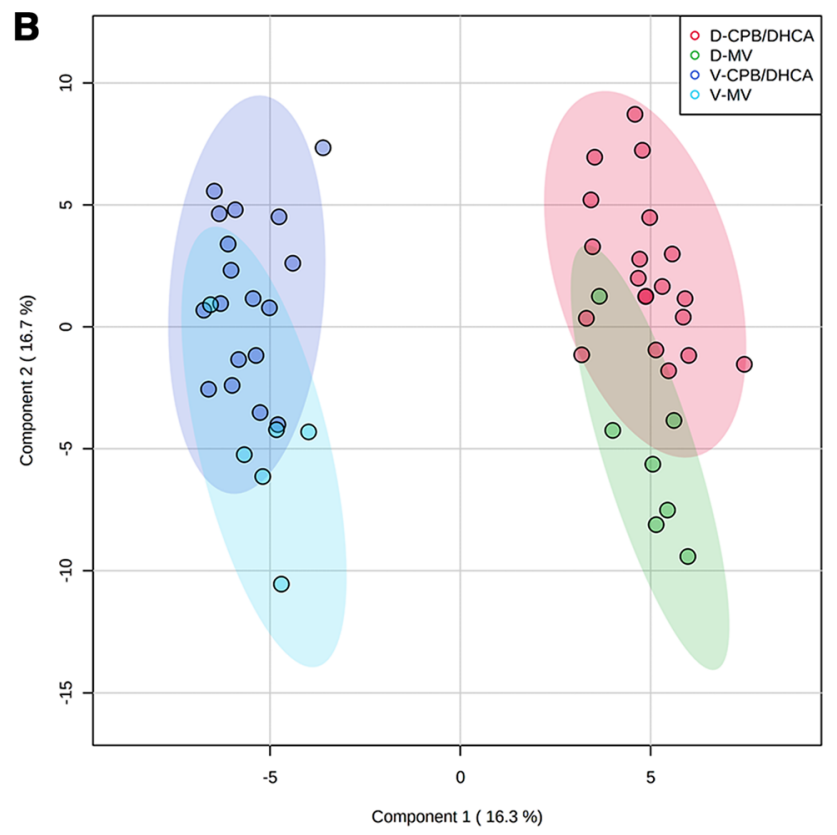

95\% confidence intervals for each group. D-CPB/DHCA Dorsocaudal region cardiopulmonary bypass and deep hypothermic circulatory arrest (CPB/DHCA), $D-M V$ Dorsocaudal region mechanical ventilation (MV), $V-C P B / D H C A$ Ventral region CPB/DHCA, $V-M V$ Ventral region $\mathrm{MV}$ 
of the lung. The marked regional differences in metabolites in the lung made it difficult to determine differentiation based on exposure to CPB/DHCA. PLS-DA was therefore performed to assess if $\mathrm{CPB} / \mathrm{DHCA}$ group differentiation could be further elicited and if so, what variables contributed most to this differentiation.

PLS-DA demonstrated discrimination between ventral and dorsocaudal regions clearly along the first two components as shown in Fig. 1B. The first two components also begin to show discrimination between CPB/DHCA and MV groups. Cross-validation showed good reproducibility $(\mathrm{R} 2=0.7 ; \mathrm{Q} 2=0.59 ; p<0.0005)$. The discrimination is especially apparent in the dorsocaudal regions compared to the ventral regions.

Variable importance in projection (VIP) was then performed to identify which metabolites contributed most to the observed distribution between 4 groups. Fifty-three metabolites had a VIP score greater than 1 and thus contribute to the data clustering. See Supplemental Table S1 for a complete list of these metabolites. Twenty-five metabolites with a VIP score greater than 1.5 are shown in Fig. 2 along with a heat map of relative peak intensities in each group. To verify the statistical significance of the metabolites interpreted as contributing to discrimination by VIP and to quantitatively compare metabolites among groups, an ANOVA was performed.
Using an FDR of $<0.05,74$ significant metabolites were found. All but 1 metabolite with a VIP score greater than 1 were also shown to be significant between groups using ANOVA. To determine specific between-group differences, post-hoc testing was performed. Results are listed in Table 1, demonstrating metabolites elevated by lung region and by CPB/DHCA. Table 1 also lists metabolites that appeared to have an additive effect by both lung region and CPB/DHCA. Please refer to the supplemental Table S2 for complete detail. The following sections highlight significant metabolites and associated pathways by lung region and by $\mathrm{CPB} /$ DHCA. HMDB ID numbers for these significant metabolites are listed in supplemental Table S3.

\subsection{Metabolic differences by lung region}

As listed in Table 1, 61 metabolites differed by lung region with 38 metabolites elevated in the ventral region and 23 metabolites elevated in the dorsocaudal region. As an example of this pattern, Fig. 3A demonstrates the normalized relative intensities of glyceric acid, which was elevated in the dorsocaudal region of both MV and CPB/DHCA animals. Pathway analysis was performed which demonstrated 18 metabolic pathways that contributed to the metabolic differences between ventral and dorsocaudal regions. Pathwayst
Fig. 2 Variable importance in projection scores and heat map. Metabolites with a Variable Importance in Projection (VIP) score greater than 1.5 contributing to variation in metabolic fingerprints between groups are shown. The heat map on the right demonstrates relative intensities of each metabolite in each group. $D B$ Dorsocaudal region cardiopulmonary bypass and deep hypothermic circulatory arrest (CPB/DHCA), DM Dorsocaudal region mechanical ventilation $(\mathrm{MV}), U D P$ Uridine diphosphate, $V B$ Ventral region, $V M$ Ventral region MV
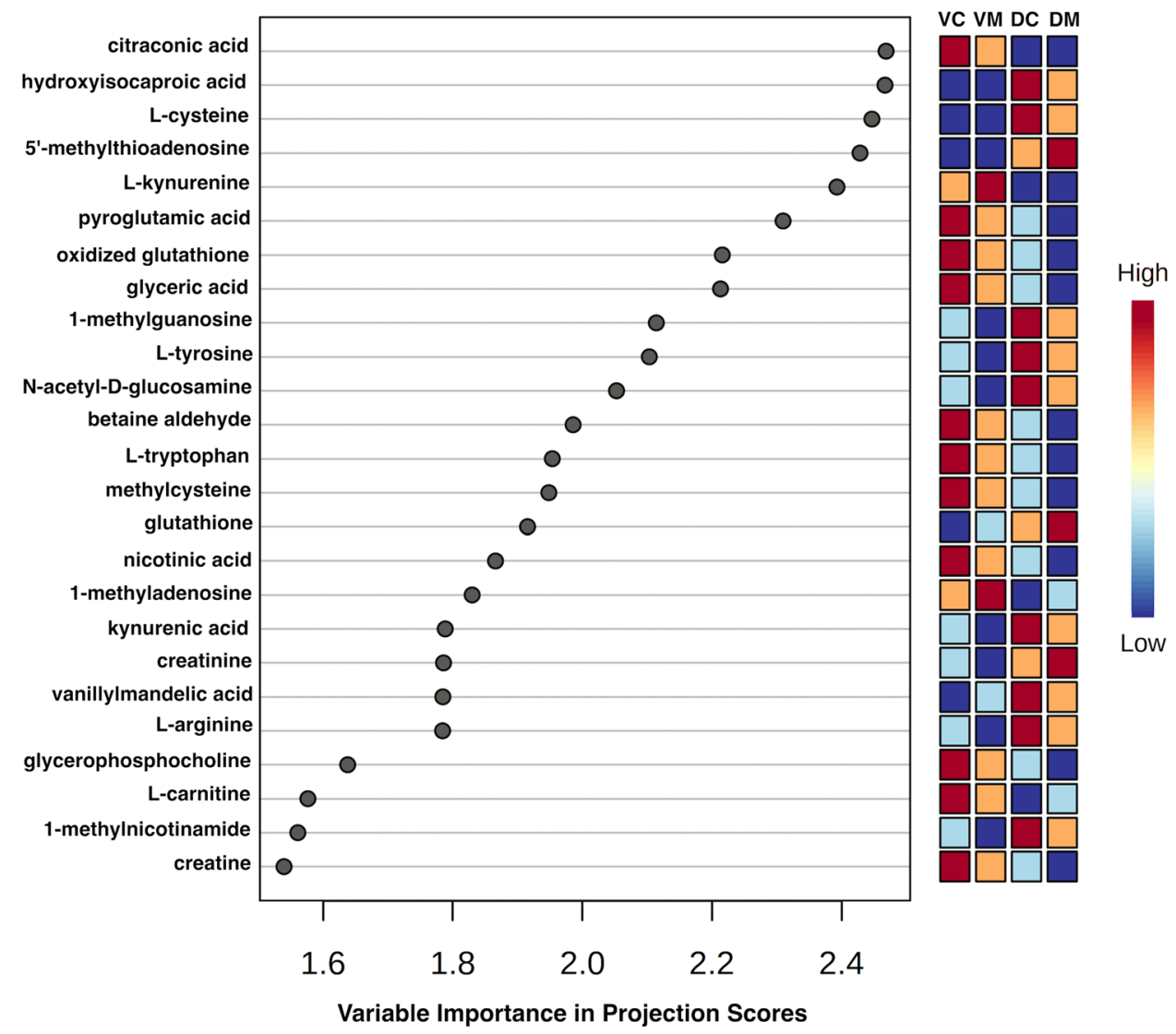
Table 1 Metabolites that differ by region and by bypass status

\begin{tabular}{|c|c|c|}
\hline Metabolites by region & Metabolites by $\mathrm{CPB} / \mathrm{DHCA}$ & Metabolites with additive effects \\
\hline Elevated in ventral region & Elevated in $C P B / D H C A$ & 2-ketobutyric acid \\
\hline 1-methylnicotinamide & 2-aminobenzoic acid & 3-phosphoglyceric acid \\
\hline 2-ketobutyric acid & 2-ketobutyric acid & 4-hydroxybenzoic acid \\
\hline 3-methylphenylacetic acid & 3-phosphoglyceric acid & Allantoin \\
\hline 3-methylthiopropionic acid & 4-Hydroxybenzoic acid & Cis-aconitic acid \\
\hline 3-phosphoglyceric acid & 4-hydroxyphenylpyruvic acid & Citric acid \\
\hline 5'-methylthioadenosine & Acetoacetic acid & Glycerophosphocholine \\
\hline 6-phosphogluconic acid & Allantoin & Isocitric acid \\
\hline Alpha-ketoisovaleric acid & Cis-aconitic acid & Kynurenic acid \\
\hline Ascorbic acid & Citric acid & L-lactic acid \\
\hline Betaine aldehyde & D-erythrose 4-phosphate & L-lysine \\
\hline Cis-aconitic acid & D-glucose & L-kynurenine \\
\hline Citric acid & Fructose-6-phosphate & Malondialdehyde \\
\hline Fumaric acid & Glycerophosphocholine & Phosphoserine \\
\hline Glucaric acid & Indoleacrylic acid & Pyruvic acid \\
\hline Glutathione & Isocitric acid & S-adenosylhomocysteine \\
\hline Hydroxyisocaproic acid & Kynurenic acid & Thiamine \\
\hline Isocitric acid & L-alanine & Ureidosuccinic acid \\
\hline Imidazoleacetic acid & L-lactic acid & \\
\hline L-asparagine & L-lysine & \\
\hline L-aspartic acid & L-proline & \\
\hline L-cysteine & Malondialdehyde & \\
\hline L-lactic acid & Pyruvic acid & \\
\hline L-malic acid & Quinolinic acid & \\
\hline L-tryptophan & S-adenosylhomocysteine & \\
\hline Maleic acid & Sedoheptulose 1,7-bisphosphate & \\
\hline $\mathrm{N}$-acetyl-D-glucosamine & Succinic acid & \\
\hline $\mathrm{N}$-acetyl-glutamate & Thiamine & \\
\hline Nicotinic acid & Ureidosuccinic acid & \\
\hline Phosphoenolpyruvic acid & Decreased in $C P B / D H C A$ & \\
\hline Phosphoserine & L-kynurenine & \\
\hline Pyrophosphate & Phosphoserine & \\
\hline Pyruvic acid & Uridine & \\
\hline \multicolumn{3}{|l|}{ S-adenosylhomocysteine } \\
\hline \multicolumn{3}{|l|}{ S-adenosylmethionine } \\
\hline \multicolumn{3}{|l|}{ Shikimate 3-phosphate } \\
\hline \multicolumn{3}{|l|}{ Thiamine } \\
\hline \multicolumn{3}{|l|}{ Trigonelline } \\
\hline \multicolumn{3}{|l|}{ Elevated in dorsocaudal region } \\
\hline \multicolumn{3}{|l|}{ 1-methyladenosine } \\
\hline 1-methylguanosine & & \\
\hline
\end{tabular}


Table 1 (continued)

\begin{tabular}{|c|c|c|}
\hline Metabolites by region & Metabolites by $\mathrm{CPB} / \mathrm{DHCA}$ & Metabolites with additive effects \\
\hline \multicolumn{3}{|l|}{ 4-hydroxybenzoic acid } \\
\hline \multicolumn{3}{|l|}{ Allantoin } \\
\hline \multicolumn{3}{|l|}{ Citraconic acid } \\
\hline \multicolumn{3}{|l|}{ Creatine } \\
\hline \multicolumn{3}{|l|}{ Creatinine } \\
\hline \multicolumn{3}{|l|}{ Glucose-6-phosphate } \\
\hline \multicolumn{3}{|l|}{ Glyceric acid } \\
\hline \multicolumn{3}{|l|}{ Glycerophosphocholine } \\
\hline \multicolumn{3}{|l|}{ Hexose phosphate ${ }^{\mathrm{a}}$} \\
\hline \multicolumn{3}{|l|}{ Kynurenic acid } \\
\hline \multicolumn{3}{|l|}{ L-arginine } \\
\hline \multicolumn{3}{|l|}{ L-carnitine } \\
\hline \multicolumn{3}{|l|}{ L-kynurenine } \\
\hline \multicolumn{3}{|l|}{ L-lysine } \\
\hline \multicolumn{3}{|l|}{ L-tyrosine } \\
\hline \multicolumn{3}{|l|}{ Malondialdehyde } \\
\hline \multicolumn{3}{|l|}{ Methylcysteine } \\
\hline \multicolumn{3}{|l|}{ Oxidized glutathione } \\
\hline \multicolumn{3}{|l|}{ Pyroglutamic acid } \\
\hline \multicolumn{3}{|l|}{ Thymine } \\
\hline Ureidosuccinic acid & & \\
\hline
\end{tabular}

Metabolites with significantly elevated relative peak intensities as determined by analysis of variance posthoc testing. Metabolites are listed by region and by cardiopulmonary bypass/deep hypothermic circulatory arrest (CPB/DHCA). Metabolites elevated in the ventral or dorsocaudal regions are listed as such. Metabolites with additive effects are those that appeared to have an additive effect of both region and CPB/DHCA. ${ }^{a}$ Hexose-phosphate represents all hexose sugar phosphates combined, as there were too many isomers to distinguish using the extraction methods used

are shown in Fig. 4A and the complete list of pathways are shown in Table 2. Please see supplemental Table S4 for more detailed statistical data. Affected pathways included those involved in the metabolism of amino acids, betaine, carbohydrates, energy, nitrogen, and vitamins. Glutathione metabolism and spermidine/spermine biosynthesis were also affected by lung region.

\subsection{Metabolic differences by CPB/DHCA}

As listed in Table 1, 29 metabolites were significantly higher in the lungs of CPB/DHCA animals. As an example of this pattern, Fig. 3B demonstrates the normalized relative intensities of acetoacetatic acid, which was elevated in both the ventral and dorsocaudal regions of CPB/DHCA animals. Pathway analysis demonstrated 2 pathways that contribute to the metabolic differences caused by CPB/DHCA alone. These included 1 pathway involved in amino acid metabolism (phenylalanine and tyrosine metabolism) and 1 pathway involved in energy metabolism (ketone body metabolism). Ketone body metabolism was unique to differences caused by CPB/DHCA while phenylalanine/tyrosine metabolism was affected by both lung region and CPB/DHCA. 

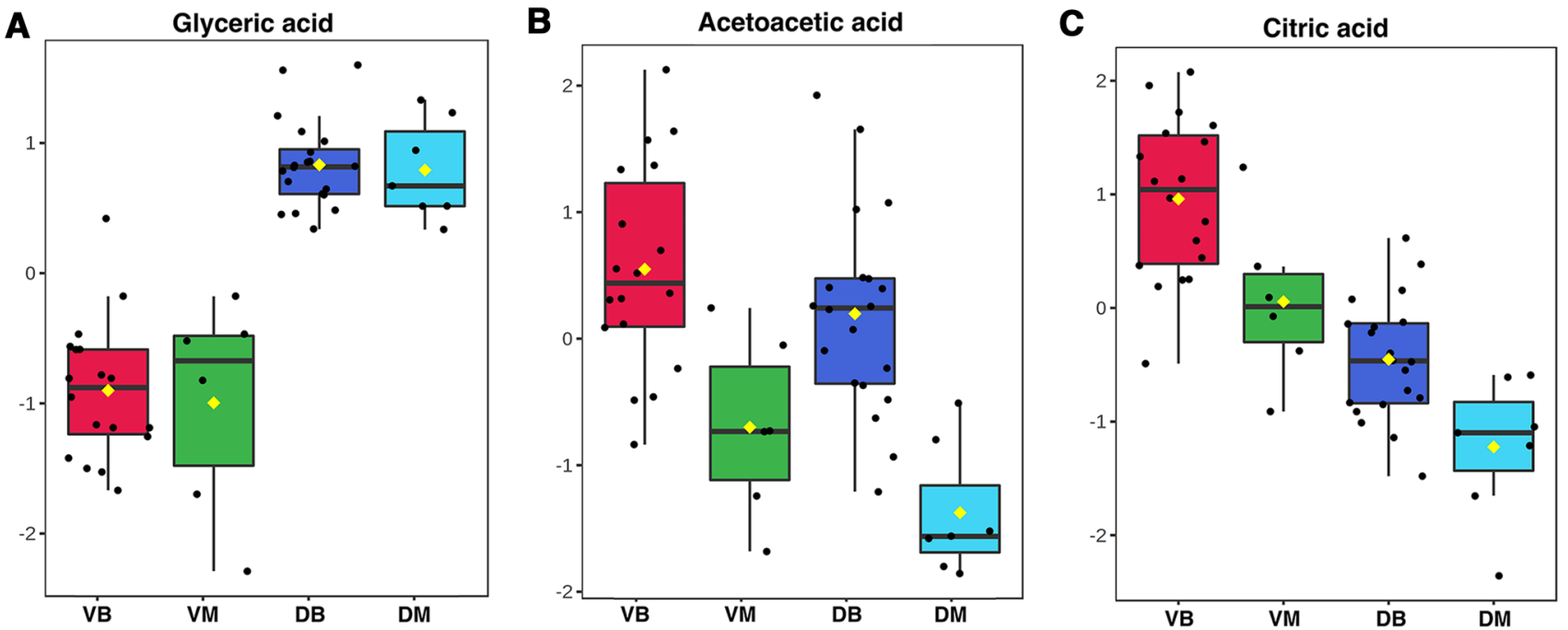

Fig. 3 Select box-plots for significant metabolites on ANOVA, Fisher FDR Post-Hoc testing. Y-axis represents transformed and scaled relative peak intensities. Yellow diamond represents the mean. A. Glyceric acid peak intensity was significantly higher in the both dorsocaudal cardiopulmonary bypass/deep hypothermic circulatory arrest (CPB/DHCA) and dorsocaudal mechanical ventilation (MV) compared to the ventral region $\mathrm{CPB} / \mathrm{DHCA}$ and MV groups $\mathbf{B}$. Acetoacetatic acid peak intensity was significantly higher in the CPB/DHCA

\subsection{Metabolites demonstrating additive effects from both lung region and CPB/DHCA}

As listed in Table 1, 18 metabolites showed additive effects from both lung region and CPB/DHCA. As an example, Fig. 3C shows the relative normalized levels of citrate, demonstrating effects of both lung region and CPB/DHCA. These 18 metabolites are involved in a number of pathways including amino acid, carbohydrate, lipid, propanoate, pyrimidine, and vitamin metabolism as well as reduction-oxidation reactions and energy production.

\section{Discussion}

\subsection{Key findings}

Using a large animal model of CPB/DHCA, we performed targeted metabolomic profiling to directly investigate the influence of CPB/DHCA on the global and regional metabolic changes in porcine lung tissue. Our results demonstrate a dramatic difference in the metabolic fingerprint of the dorsocaudal lung region compared to the ventral lung region in both animals that underwent $\mathrm{CPB} / \mathrm{DHCA}$ and control animals that underwent MV. To a lesser extent, lung tissue from the CPB/DHCA animals showed a unique metabolic fingerprint compared to MV animals. Key drivers of these metabolic changes were metabolites involved in essential groups compared to MV groups $\mathbf{C}$. Citric acid peak intensity is significantly higher in both the ventral regions compared to dorsocaudal regions and also with $\mathrm{CPB} / \mathrm{DCHA}$ compared to MV. It also shows an additive effect in that the relative peak intensity is significantly elevated in the ventral region CPB/DHCA group compared to all the other groups. $D B$ Dorsocaudal CPB/DHCA, $D M$ Dorsocaudal MV, $V B$ Ventral CPB/DHCA, $V M$ Ventral MV

cell function pathways including energy, carbohydrate, and nitrogen metabolism, amino acid synthesis and degradation, vitamin metabolism, and reduction-oxidation reactions. Due to the abundance of significant results that have important prognostic and therapeutic potential, a comprehensive review of all pathways is beyond the scope of this article. Specific pathways will be discussed based on their statistical significance, contribution to the understanding of injury mechanisms, and potential to be therapeutic targets.

\subsection{Isolated regional differences in lung tissue metabolic fingerprint}

The metabolic fingerprint of the dorsocaudal lung samples differed dramatically from the ventral lung samples in both control animals and the CPB/DHCA group, with the majority of pathways demonstrating additive effects by lung region and $\mathrm{CPB} / \mathrm{DHCA}$ exposure (discussed in subsequent sections). Several metabolic pathways appear to be influenced primarily by lung region, with the most notable being glutathione (GSH) metabolism. Our findings suggest a difference in oxidative states between lung regions, with elevation of upstream reactants GSH and L-cysteine in the ventral regions and elevation of oxidized glutathione (GSSG) and pyroglutamic acid in the dorsocaudal regions. Other studies have shown that the balance among GSH, L-cysteine, and GSSG are altered in BALF and serum of humans with lung injury (Bernard et al., 1997; Kellner et al., 


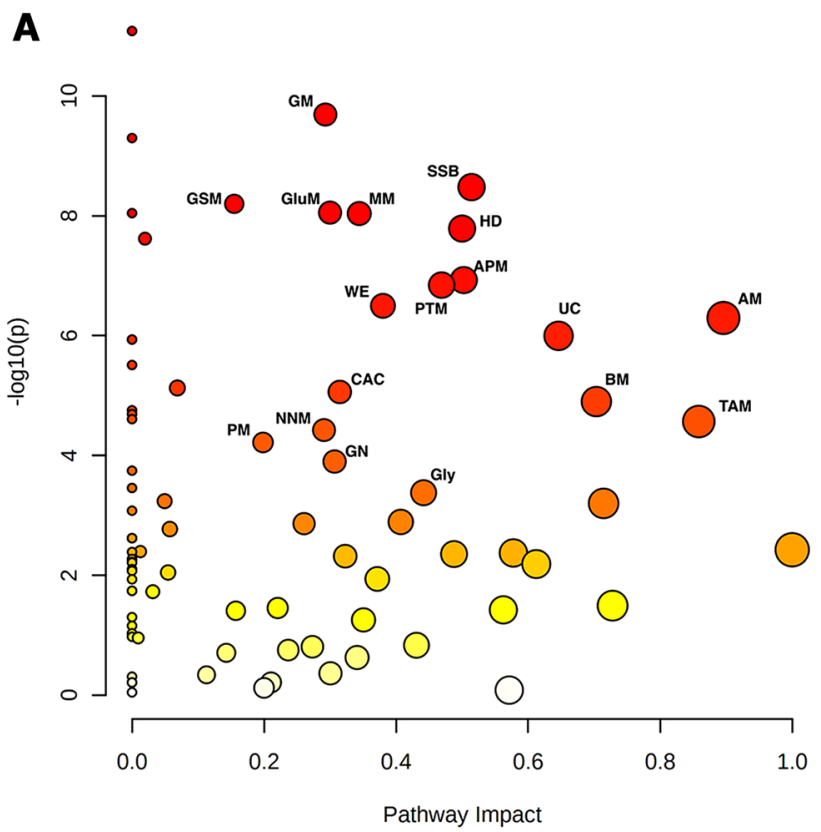

Fig. 4 Pathway analysis. The $\mathrm{x}$-axis reflects the impact factor calculated from Topographic Analysis; the y-axis reflects the negative logarithm of the $p$-value calculated from the Metabolite Set Enrichment Analysis. Each circle reflects a pathway. Node size reflects the impact value and node color reflects the $\mathrm{p}$-value with lower $p$-values displayed as red and higher $p$-values displayed as more yellow-white. Select pathways are labeled. A. Pathways contributing to regional differences. B. Pathways contributing to cardiopulmonary bypass/deep hypothermic circulatory arrest differences (CPB/DHCA). AM Aspar-

2017), and our model adds to these findings by identifying the dorsocaudal lung as the potential source of GSSG. This finding has important translational implications as GSH metabolism is being investigated for its therapeutic potential in lung diseases. For example, repletion of both GSH with the precursor $\mathrm{N}$-acetylcysteine (NAC) and L-cysteine with precursor pro-cysteine decreases the duration of ALI in human subjects (Bernard et al., 1997). In mice undergoing $\mathrm{CPB} / \mathrm{DHCA}$, increasing glutathione peroxidase activity reduced markers of multi-organ damage and ischemia/ reperfusion injury in the lung (Steinbrenner et al., 2016). GSH supplementation has also been used to treat dyspnea in patients with COVID-19 (Horowitz et al., 2020). Our findings of regional oxidation of glutathione in the dorsocaudal lung suggest that increased oxidative stress in the posterior lung of supine patients could contribute to the increased lung injury seen in this area. Given the promising uses of GSH-related therapy for inflammation-induced lung injury, targeted GSH or other antioxidant therapy to the posterior lung could represent a novel mitigation strategy for postCPB lung inflammation.

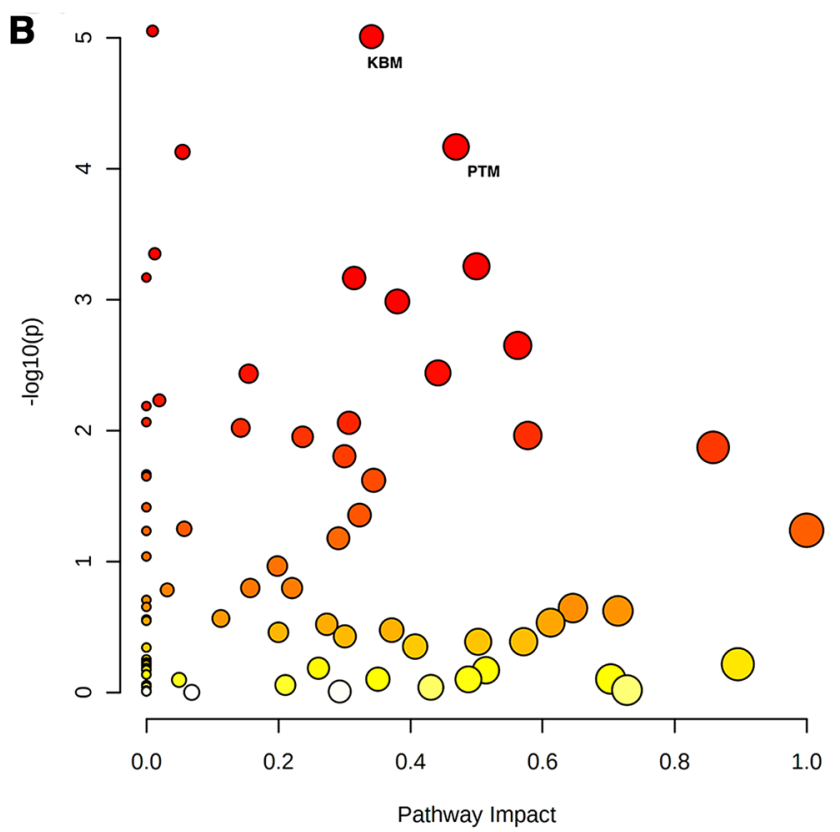

tate metabolism, APM Arginine and proline metabolism, $B M$ Betaine metabolism, $C A C$ Citric acid cycle, GluM Glutamate metabolism, Gly Glycolysis, GM Glutathione metabolism, GN Gluconeogenesis, GSM Glycine and serine metabolism, $H D$ Homocysteine degradation, $K B M$ Ketone body metabolism, $M M$ Methionine metabolism, $N N M$ Nicotinate and nicotinamide metabolism, PM Pyruvate metabolism, PTM Phenylalanine and tyrosine metabolism, SSB Spermine and spermidine biosynthesis, $U C$ Urea cycle, WE Warburg effect

\subsection{Isolated CPB/DHCA effects on lung metabolome}

CPB/DHCA also influenced the lung metabolome, but to a lesser extent than lung region. The main pathway that showed significant effects from $\mathrm{CPB} / \mathrm{DHCA}$ exposure but not from lung region was ketone body metabolism. Specifically, we found that CPB/DHCA significantly altered ketone body metabolism on pathway analysis and the ketone body acetoacetic acid was elevated in CPB/DHCA compared to $\mathrm{MV}$. Although the liver is known to be the major source of ketone bodies following CPB/DHCA (Nomoto et al., 1992; Ozawa et al., 1983; Poggetti et al., 1992), the lungs themselves also have the potential to produce ketone bodies. The lungs use fatty acid beta oxidation during stress states, leading to ketone body production (Liu \& Summer, 2019). Furthermore, unique isotypes of the rate limiting enzyme for ketone body production, HMG-CoA lyase, exist in the lung (Arnedo et al., 2019) but thus far there are limited studies on their role in lung injury. Our study is the first to suggest that the lungs may participate in ketone body synthesis following CPB/DHCA. As ketone body metabolism plays a protective role in stress states (Arnedo et al., 2019), and is being proposed as a potential therapy to prevent ARDS in patients with COVID-19 (Bradshaw et al., 2020), our findings should 
Table 2 Pathway analysis

\begin{tabular}{|c|c|c|c|c|}
\hline \multicolumn{2}{|l|}{ Pathways affected by lung region } & \multirow{2}{*}{$\begin{array}{l}\text { Matched metabolites/ } \\
\text { total metabolites }\end{array}$} & \multirow[t]{2}{*}{$P$-value } & \multirow[t]{2}{*}{ Impact } \\
\hline Category & Pathway & & & \\
\hline \multirow[t]{7}{*}{ Amino acid metabolism } & Arginine and proline metabolism & $18 / 48$ & $1.18 \mathrm{E}-07$ & 0.50259 \\
\hline & Aspartate metabolism & $13 / 34$ & 5.07E-07 & 0.89583 \\
\hline & Glutamate metabolism & $16 / 45$ & $8.86 \mathrm{E}-09$ & 0.29996 \\
\hline & Glycine and serine metabolism & $21 / 50$ & $6.32 \mathrm{E}-09$ & 0.15486 \\
\hline & Homocysteine degradation & $5 / 7$ & $1.63 \mathrm{E}-08$ & 0.5 \\
\hline & Methionine metabolism & $18 / 39$ & $9.17 \mathrm{E}-09$ & 0.34418 \\
\hline & Phenylalanine and tyrosine metabolism & $9 / 25$ & $1.43 \mathrm{E}-07$ & 0.46903 \\
\hline Betaine metabolism & Betaine metabolism & $10 / 18$ & $1.26 \mathrm{E}-05$ & 0.70336 \\
\hline \multirow[t]{2}{*}{ Carbohydrate metabolism } & Gluconeogenesis & $13 / 30$ & $1.26 \mathrm{E}-04$ & 0.30686 \\
\hline & Glycolysis & $9 / 20$ & 4.18E-04 & 0.44167 \\
\hline \multirow[t]{4}{*}{ Energy metabolism } & Citric acid cycle & $11 / 26$ & $8.70 \mathrm{E}-06$ & 0.3147 \\
\hline & Pyruvate metabolism & $9 / 37$ & $6.06 \mathrm{E}-05$ & 0.19838 \\
\hline & Transfer of acetyl groups into mitochondria & $6 / 18$ & $2.71 \mathrm{E}-05$ & 0.85846 \\
\hline & Warburg effect & $19 / 49$ & $3.18 \mathrm{E}-07$ & 0.3801 \\
\hline Nitrogen metabolism & Urea cycle & $13 / 23$ & $1.01 \mathrm{E}-06$ & 0.64611 \\
\hline Reduction-oxidation reactions & Glutathione metabolism & $5 / 19$ & $2.04 \mathrm{E}-10$ & 0.29287 \\
\hline Spermidine and spermine biosynthesis & Spermidine and spermine biosynthesis & $5 / 14$ & 3.32E-09 & 0.51429 \\
\hline Vitamin metabolism & Nicotinate and nicotinamide metabolism & $8 / 32$ & $3.77 \mathrm{E}-05$ & 0.29083 \\
\hline \multicolumn{2}{|l|}{ Pathways affected by $C P B / D H C A$} & \multirow{2}{*}{$\begin{array}{l}\text { Matched metabolites/ } \\
\text { total metabolites }\end{array}$} & \multirow[t]{2}{*}{$P$-value } & \multirow[t]{2}{*}{ Impact } \\
\hline Category & Pathway & & & \\
\hline Amino acid metabolism & Phenylalanine and tyrosine metabolism & $9 / 25$ & $6.82 \mathrm{E}-05$ & 0.46903 \\
\hline Energy metabolism & Ketone body metabolism & $2 / 12$ & $9.82 \mathrm{E}-06$ & 0.34091 \\
\hline
\end{tabular}

Significant pathways contributing to regional effects and cardiopulmonary bypass/deep hypothermic circulatory arrest (CPB/DHCA) are shown. Matched metabolites/Total metabolites reflects the number of metabolites that were sampled out of the total number of metabolites in given pathway. $P$-value was calculated by Metabolite Set Enrichment Analysis and Impact Factor was calculated by Topographic Analysis. FDR False Discovery Rate. Adjusted P-value P-value adjusted by the Holm-Bonferonni correction

be further investigated to evaluate the prognostic and therapeutic applications of ketone body metabolism in $\mathrm{CPB} /$ DHCA patients.

While our study is the first to examine tissue-level metabolic changes after $\mathrm{CPB}$, Maltesen et al. did examine the effects of CPB on metabolomic changes in BALF from a cohort of adults with COPD who were undergoing cardiac surgery (Maltesen et al., 2018). The authors showed similar metabolomic changes to those found in our study, namely elevated metabolites involved in glycolysis, urea cycle, protein catabolism, urea metabolism, purine metabolism, and nitric oxide synthesis. It is unclear how much lung region could have contributed to these findings as the sampling of BALF in this study was obtained only from the right middle lobe. Furthermore, Maltesen's study used adult patients with the confounding variable of chronic lung disease. These limitations demonstrate the importance of using complementary, clinically relevant translational models to study the complex metabolic changes in cardiac surgery patients. Specifically, pediatric porcine models may more accurately reflect pediatric congenital heart surgery patients, since pigs have similar lung metabolome, anatomy, histology, and immune systems, and thus serve as good models of humans in physiological and mechanistic studies (Judge et al., 2014; Mair et al., 2014).

\subsection{Additive effects of CPB/DHCA and lung region}

Lung region and CPB/DHCA together also have substantial additive effects on specific metabolites and associated pathways. Glycolysis, the citric acid cycle (CAC), and the tryptophan-kynurenine pathway are all affected in an additive fashion and demonstrate the importance of considering lung region in the investigation of $\mathrm{CPB} / \mathrm{DHCA}$ effects on the lung metabolome. For example, in the ventral region but not dorsocaudal region CPB/DHCA may have increased energy producing glycolytic and CAC flux based on elevation of 3-phosphoglyceric acid (a metabolite in the energy generating pathway of glycolysis) (Liu \& Summer, 2019), and pyruvate (the end product of aerobic glycolysis which is 
then either converted into lactate or enters the CAC) (Liu \& Summer, 2019). Lactate and the first three metabolites of the CAC (citric acid, cis-aconitic acid, and isocitrate) were also elevated in both the ventral region and in the CPB/DHCA group. The study by Maltesen et al., also showed alterations in glucose and energy metabolism with CPB (Maltesen et al., 2018), and our study demonstrating that these effects are focused regionally. A study by Eckle et al. further illustrates the importance of considering regional lung metabolome analysis in ALI. The authors showed that in murine MV-induced ALI, hypoxia inducible factors (HIF) were stabilized by alveolar distension, leading to succinate dehydrogenase inhibition, alteration of TCA flux, and stabilization of mitochondrial glycolytic capacity (Eckle et al., 2013). Gravity independent regions have comparatively more ventilation and alveolar distension with less atelectasis than gravity dependent regions (Carvalho et al., 1985; Magnusson et al., 1997; West, 2011). Thus, Eckle's study shows a potential mechanism for the gravity dependent metabolic changes in a lung with a potentially injurious stimulus. There are therapeutic implications for this as well as HIFs are being studied as therapeutic targets for a number of conditions including both ALI and pulmonary hypertension (Lee et al., 2019).

Metabolites of the tryptophan-kynurenine pathway also showed variance based on both $\mathrm{CPB} / \mathrm{DHCA}$ and lung region as tryptophan was elevated in the ventral lung, while downstream metabolites kynurenine (KYN) and kynurenic acid (KYNA) were in both elevated in the dorsocaudal lung and both altered CPB/DHCA animals. This finding demonstrates potential regional lung sources of KYN and KYNA, both of which have been demonstrated by our group to be elevated in serum from infants undergoing CPB (Davidson et al., 2018). Serum KYNA is associated with worse outcomes in infants following CPB/DHCA for heart surgery (Davidson et al., 2018). As KYN has potent vasodilatory effects on lung vasculature (Nagy et al., 2017; Wang et al., 2015) and the KYN arm of the tryptophan-kynurenine pathway plays important roles in oxidative injury (Lugo-Huitrón et al., 2011; Wang et al., 2015), multiple drugs are under development that target KYN pathway enzymes to modulate inflammation and oxidative injury for cancer and neurodegenerative diseases (Dounay et al., 2015). The availability of these drugs along with the data supplied by the current study that help localize sources of these metabolites are promising for the future of research investigating KYN pathway modulators for treatment of CPB-related lung injury.

\subsection{Limitations}

This study has several limitations. First, the small number of animals, particularly in the MV group, limited the power of the study; a larger sample size may have yielded more significant metabolites and pathways. This especially was likely to contribute to the low numbers of statistically significant pathways observed when comparing CPB/DHCA vs MV. Similarly, the study lacked a control group that was unexposed to MV. Therefore, we can only comment on regional differences under mechanical ventilation as opposed to the effects of mechanical ventilation compared to spontaneous breathing. Next, the injury was assessed at a single time point, early in the postoperative course. Systemic inflammation and pulmonary dysfunction following CPB/DHCA evolves from the time of CPB/DHCA initiation to $48 \mathrm{~h}$ post-surgery with peak inflammation typically between $3 \mathrm{~h}$ to $12 \mathrm{~h}$ following CPB/DHCA. Thus, studies exploring serial time points may more completely reflect an operative and post-operative course and help capture the most accurate metabolic alterations. Also, due to their similar lung metabolome, anatomy, histology, and immune systems, pigs are known to serve as good models of humans in physiological and mechanistic studies (Benahmed et al., 2014; Judge et al., 2014; Mair et al., 2014). They are not, however, perfect substitutes for humans in clinical trials. Another important limitation is our study used peripheral cannulation, while midline sternotomy with central cannulation is typically used in humans for CPB/DHCA. While this strategy allowed us to isolate the effects of CPB/DHCA from the mechanical effects of an open thorax, it is possible that our model could underrepresent the full extent of injury seen during surgery.

In terms of pathway analysis, pathways were grouped based on similar features to better describe patterns of pathway disturbances. This method, however, may lead to missed pathway categories, overlapping categories, or poorly fit pathways into categories. Lastly, many of the significant metabolites are involved in anapleurotic reactions, meaning they are used in several different metabolic pathways. This makes it difficult to draw mechanistic conclusions; thus, results from this study should be used to generate more targeted studies on individual metabolites, fully mapped metabolic pathways, and the activity of associated enzymes. Also, some metabolites may have different redox stability, such as GSH and GSSH, but processing and analysis of all samples was done uniformly and simultaneously. To best move forward from these limitations and elaborate on the current study, there are several exciting research methods to pursue: tissue metabolic pathways can be further explored with paired analysis of tissue and local arterial/venous blood sampling. Exploring structure-function relationships can accomplished with sub-regional, cellular, or sub-cellular analysis. Pathway modulation and flux studies could further describe mechanisms of metabolic pathway alterations. Taken together, these future studies could be used to design novel biomarkers and therapies for clinical application. 


\section{Overall conclusions}

The current study was the first to directly analyze the metabolome of lung tissue following CPB/DHCA and compare the metabolome of ventral and dorsocaudal lung regions. The results identified a large number of significantly altered metabolites and metabolic pathways, demonstrating broad metabolic disturbances in gravity dependent lung regions during supine mechanical ventilation with or without $\mathrm{CPB} /$ DHCA. Exposure to CPB/DHCA also led to metabolic differences and often had additive effects to the regional disturbances. Most perturbed pathways included the metabolism of amino acids, carbohydrates, lipids, steroids, vitamins, and reduction-oxidation pathways.

Supplementary Information The online version contains supplementary material available at https://doi.org/10.1007/s11306-021-01842-y.

Acknowledgements We would like to thank the team in the University of Colorado Office of Laboratory Animal Resources for the many long days assisting with the surgeries for this study.

Author contributions SJC drafted the article. SJC, LK, JD designed and conceptualized the study. SJC, LK, BF, JD performed data analysis and interpretation. JK developed the metabolite analysis methods and acquired the data. JK and LK analyzed the samples. LK, JR, SO, and JD performed the animal surgery. JR, JJ, RI, SL, SO, and JD developed the model. SO and JD acquired funding. JD mentored the preparation of the manuscript. All authors critically revised the article and approved the article for submission.

Funding This study was supported by Department of Defense PR152240 (PI Davidson), American Heart Association 17IRG33410724 (PI Davidson), and National Institutes of Health/ National Heart, Lung, and Blood Institute K23HL123634 (PI Davidson).

Data availability The metabolomics and metadata reported in this paper are available in the online data supplement.

Code availability Not applicable.

\section{Declarations}

Conflict of interest No authors declare any conflict of interest.

Ethical approval The animal protocol used was approved by the Institutional Animal Care and Use Committee of the University of Colorado (Protocol Number: 107715(02)1D) in accordance with the Guide for the Care and Use of Laboratory Animals and the ARRIVE guidelines.

Animal rights All applicable international, national, and/or institutional guidelines for the care and use of animals were followed.

Consent to participate Not applicable.

Consent for publication Not applicable.

\section{References}

Altemeier, W. A., McKinney, S., Krueger, M., \& Glenny, R. W. (1985). (2004) Effect of posture on regional gas exchange in pigs. Journal of Applied Physiology, 97, 2104-2111.

Apostolakis, E., Filos, K. S., Koletsis, E., \& Dougenis, D. (2010). Lung dysfunction following cardiopulmonary bypass. Journal of Cardiac Surgery, 25, 47-55.

Arnedo, M., Latorre-Pellicer, A., Lucia-Campos, C., Gil-Salvador, M., Antoñanzas-Peréz, R., Gómez-Puertas, P., Bueno-Lozano, G., Puisac, B., \& Pié, J. (2019). More than one HMG-CoA lyase: The classical mitochondrial enzyme plus the peroxisomal and the cytosolic ones. International Journal of Molecular Science, 20.

Benahmed, M. A., Elbayed, K., Daubeuf, F., Santelmo, N., Frossard, N., \& Namer, I. J. (2014). NMR HRMAS spectroscopy of lung biopsy samples: Comparison study between human, pig, rat, and mouse metabolomics. Magnetic Resonance in Medicine, 71, $35-43$.

Benjamin, E. J., Virani, S. S., Callaway, C. W., Chamberlain, A. M., Chang, A. R., Cheng, S., Chiuve, S. E., Cushman, M., Delling, F. N., Deo, R., de Ferranti, S. D., Ferguson, J. F., Fornage, M., Gillespie, C., Isasi, C. R., Jiménez, M. C., Jordan, L. C., Judd, S. E., Lackland, D., ... Muntner, P. (2018). Heart disease and stroke statistics-2018 update: A report from the american heart association. Circulation, 137, e67-e492.

Bernard, G. R., Wheeler, A. P., Arons, M. M., Morris, P. E., Paz, H. L., Russell, J. A., \& Wright, P. E. (1997). A trial of antioxidants $\mathrm{N}$-acetylcysteine and procysteine in ARDS. The Antioxidant in ARDS Study Group. Chest, 112, 164-172.

Bowler, R. P., Wendt, C. H., Fessler, M. B., Foster, M. W., Kelly, R. S., Lasky-Su, J., Rogers, A. J., Stringer, K. A., Winston, B. W., \& American Thoracic Society Workgroup on, M. and Proteomics. (2017). New strategies and challenges in lung proteomics and metabolomics. An official American thoracic society workshop report. Annals of the American Thoracic Society, 14, 1721-1743.

Bradshaw, P. C., Seeds, W. A., Miller, A. C., Mahajan, V. R., \& Curtis, W. M. (2020). COVID-19: Proposing a ketone-based metabolic therapy as a treatment to blunt the cytokine storm. Oxidative Medicine and Cellular Longevity, 2020, 6401341.

Carvalho, A. R., Spieth, P. M., Guldner, A., Cuevas, M., Carvalho, N. C., Beda, A., Spieth, S., Stroczynski, C., Wiedemann, B., Koch, T., Pelosi, P., \& de Abreu, M. G. (1985). (2011) Distribution of regional lung aeration and perfusion during conventional and noisy pressure support ventilation in experimental lung injury. Journal of Applied Physiology, 110, 1083-1092.

Chong, J., Wishart, D. S., \& Xia, J. (2019). Using MetaboAnalyst 4.0 for comprehensive and integrative metabolomics data analysis. Current Protocols in Bioinformatics, 68, e86.

Davidson, J. A., Khailova, L., Treece, A., Robison, J., Soranno, D. E., Jaggers, J., Ing, R. J., Lawson, S., \& Lujan, S. O. (2019). Alkaline phosphatase treatment of acute kidney injury in an infant piglet model of cardiopulmonary bypass with deep hypothermic circulatory arrest. Science and Reports, 9, 14175.

Davidson, J. A., Pfeifer, Z., Frank, B., Tong, S., Urban, T. T., Wischmeyer, P. A., Mourani, P., Landeck, B., Christians, U., \& Klawitter, J. (2018). Metabolomic fingerprinting of infants undergoing cardiopulmonary bypass: Changes in metabolic pathways and association with mortality and cardiac intensive care unit length of stay. Journal of the American Heart Association, 7, e010711.

Dounay, A. B., Tuttle, J. B., \& Verhoest, P. R. (2015). Challenges and opportunities in the discovery of new therapeutics targeting the kynurenine pathway. Journal of Medicinal Chemistry, 58, 8762-8782.

Eckle, T., Brodsky, K., Bonney, M., Packard, T., Han, J., Borchers, C. H., Mariani, T. J., Kominsky, D. J., Mittelbronn, M., \& Eltzschig, 
H. K. (2013). HIF1A reduces acute lung injury by optimizing carbohydrate metabolism in the alveolar epithelium. PLoS Biol, 11, e1001665.

Freitas, C. R., Malbouisson, L. M., Benicio, A., Negri, E. M., Bini, F. M., Massoco, C. O., Otsuki, D. A., Melo, M. F., \& Carmona, M. J. (2016). Lung perfusion and ventilation during cardiopulmonary bypass reduces early structural damage to pulmonary parenchyma. Anesthesia and Analgesia, 122, 943-952.

Frolkis, A., Knox, C., Lim, E., Jewison, T., Law, V., Hau, D. D., Liu, P., Gautam, B., Ly, S., Guo, A. C., Xia, J., Liang, Y., Shrivastava, S., \& Wishart, D. S. (2010). SMPDB: The small molecule pathway database. Nucleic Acids Research, 38, D480-D487.

Horowitz, R. I., Freeman, P. R., \& Bruzzese, J. (2020). Efficacy of glutathione therapy in relieving dyspnea associated with COVID19 pneumonia: A report of 2 cases. Respiratory Medicine Case Reports, 30, 101063.

Hummel, M., Meister, R., \& Mansmann, U. (2007). GlobalANCOVA: Exploration and assessment of gene group effects. Bioinformatics, 24, 78-85.

Jewison, T., Su, Y., Disfany, F. M., Liang, Y., Knox, C., Maciejewski, A., Poelzer, J., Huynh, J., Zhou, Y., Arndt, D., Djoumbou, Y., Liu, Y., Deng, L., Guo, A. C., Han, B., Pon, A., Wilson, M., Rafatnia, S., Liu, P., \& Wishart, D. S. (2014). SMPDB 2.0: Big improvements to the small molecule pathway database. Nucleic Acids Research, 42, D478-D484.

Johnson, C. H., Ivanisevic, J., \& Siuzdak, G. (2016). Metabolomics: Beyond biomarkers and towards mechanisms. Nature Reviews Molecular Cell Biology, 17, 451-459.

Judge, E. P., Hughes, J. M., Egan, J. J., Maguire, M., Molloy, E. L., \& O'Dea, S. (2014). Anatomy and bronchoscopy of the porcine lung. A model for translational respiratory medicine. American Journal of Respiratory Cell and Molecular Biology, 51, 334-343.

Kellner, M., Noonepalle, S., Lu, Q., Srivastava, A., Zemskov, E., \& Black, S. M. (2017). ROS Signaling in the pathogenesis of Acute Lung Injury (ALI) and Acute Respiratory Distress Syndrome (ARDS). Advances in Experimental Medicine and Biology, 967, 105-137.

Khemani, R. G., Smith, L., Lopez-Fernandez, Y. M., Kwok, J., Morzov, R., Klein, M. J., Yehya, N., Willson, D., Kneyber, M. C. J., Lillie, J., Fernandez, A., Newth, C. J. L., Jouvet, P., \& Thomas, N. J. (2019). Paediatric acute respiratory distress syndrome incidence and epidemiology (PARDIE): An international, observational study. The Lancet Respiratory Medicine, 7, 115-128.

Kozik, D. J., \& Tweddell, J. S. (2006). Characterizing the inflammatory response to cardiopulmonary bypass in children. Annals of Thoracic Surgery, 81, S2347-S2354.

Lee, J. W., Ko, J., Ju, C., \& Eltzschig, H. K. (2019). Hypoxia signaling in human diseases and therapeutic targets. Experimental \& Molecular Medicine, 51,1-13.

Liu, G., \& Summer, R. (2019). Cellular metabolism in lung health and disease. Annual Review of Physiology, 81, 403-428.

Liu, X., Chen, Q., Shi, S., Shi, Z., Lin, R., Tan, L., Yu, J., Shu, Q., \& Fang, X. (2012). Plasma sRAGE enables prediction of acute lung injury after cardiac surgery in children. Critical Care, 16, R91.

Lugo-Huitrón, R., Blanco-Ayala, T., Ugalde-Muñiz, P., Carrillo-Mora, P., Pedraza-Chaverrí, J., Silva-Adaya, D., Maldonado, P. D., Torres, I., Pinzón, E., Ortiz-Islas, E., López, T., García, E., Pineda, B., Torres-Ramos, M., Santamaría, A., \& La Cruz, V. P. (2011). On the antioxidant properties of kynurenic acid: Free radical scavenging activity and inhibition of oxidative stress. Neurotoxicology and Teratology, 33, 538-547.

Magnusson, L., Zemgulis, V., Wicky, S., Tyden, H., Thelin, S., \& Hedenstierna, G. (1997). Atelectasis is a major cause of hypoxemia and shunt after cardiopulmonary bypass: An experimental study. Anesthesiology, 87, 1153-1163.
Mair, K. H., Sedlak, C., Käser, T., Pasternak, A., Levast, B., Gerner, W., Saalmüller, A., Summerfield, A., Gerdts, V., Wilson, H. L., \& Meurens, F. (2014). The porcine innate immune system: An update. Developmental and Comparative Immunology, 45, 321-343.

Maltesen, R. G., Buggeskov, K. B., Andersen, C. B., Plovsing, R., Wimmer, R., Ravn, H. B., \& Rasmussen, B. S. (2018) Lung protection strategies during cardiopulmonary bypass affect the composition of bronchoalveolar fluid and lung tissue in cardiac surgery patients. Metabolites, 8 .

Matute-Bello, G., Downey, G., Moore, B. B., Groshong, S. D., Matthay, M. A., Slutsky, A. S., \& Kuebler, W. M. (2011). An official American Thoracic Society workshop report: Features and measurements of experimental acute lung injury in animals. American Journal of Respiratory Cell and Molecular Biology, 44, 725-738.

Mussap, M., Antonucci, R., Noto, A., \& Fanos, V. (2013). The role of metabolomics in neonatal and pediatric laboratory medicine. Clinica Chimica Acta, 426, 127-138.

Nagy, B. M., Nagaraj, C., Meinitzer, A., Sharma, N., Papp, R., Foris, V., Ghanim, B., Kwapiszewska, G., Kovacs, G., Klepetko, W., Pieber, T. R., Mangge, H., Olschewski, H., \& Olschewski, A. (2017). Importance of kynurenine in pulmonary hypertension. American Journal of Physiology. Lung Cellular and Molecular Physiology, 313, L741-1751.

Nomoto, S., Shimahara, Y., Kumada, K., Ogino, H., Okamoto, Y., \& Ban, T. (1992). Arterial ketone body ratio during and after cardiopulmonary bypass. Journal of Thoracic and Cardiovascular Surgery, 103, 1164-1167.

Ozawa, K., Aoyama, H., Yasuda, K., Shimahara, Y., Nakatani, T., Tanaka, J., Yamamoto, M., Kamiyama, Y., \& Tobe, T. (1983). Metabolic abnormalities associated with postoperative organ failure: A redox theory. Archives of Surgery, 118, 1245-1251.

Pasquali, S. K., He, X., Jacobs, M. L., Shah, S. S., Peterson, E. D., Gaies, M. G., Hall, M., Gaynor, J. W., Hill, K. D., Mayer, J. E., Li, J. S., \& Jacobs, J. P. (2014). Excess costs associated with complications and prolonged length of stay after congenital heart surgery. Annals of Thoracic Surgery, 98, 1660-1666.

Poggetti, R. S., Moore, F. A., Moore, E. E., Bensard, D. D., Anderson, B. O., \& Banejee, A. (1992). Liver injury is a reversible neutrophil-mediated event following gut ischemia. Archives of Surgery, 127, 175-179.

Shi, S., Chen, C., Zhao, D., Liu, X., Cheng, B., Wu, S., Lin, R., Tan, L., Fang, X., \& Shu, Q. (2014). The role of plasma gelsolin in cardiopulmonary bypass induced acute lung injury in infants and young children: A pilot study. BMC Anesthesiology, 14, 67.

Shi, S., Zhao, Z., Liu, X., Shu, Q., Tan, L., Lin, R., Shi, Z., \& Fang, X. (2008). Perioperative risk factors for prolonged mechanical ventilation following cardiac surgery in neonates and young infants. Chest, 134, 768-774.

Steinbrenner, H., Bilgic, E., Pinto, A., Engels, M., Wollschläger, L., Döhrn, L., Kellermann, K., Boeken, U., Akhyari, P., \& Lichtenberg, A. (2016). Selenium pretreatment for mitigation of ischemia/ reperfusion injury in cardiovascular surgery: Influence on acute organ damage and inflammatory response. Inflammation, 39, 1363-1376.

Stephens, R. S., Shah, A. S., \& Whitman, G. J. (2013). Lung injury and acute respiratory distress syndrome after cardiac surgery. Annals of Thoracic Surgery, 95, 1122-1129.

Tojo, K., Nagamine, Y., Yazawa, T., Mihara, T., Baba, Y., Ota, S., Goto, T., \& Kurahashi, K. (2015). Atelectasis causes alveolar hypoxia-induced inflammation during uneven mechanical ventilation in rats. Intensive Care Medicine Experimental, 3, 56.

Wang, Q., Liu, D., Song, P., \& Zou, M. H. (2015). Tryptophan-kynurenine pathway is dysregulated in inflammation, and immune activation. Front Biosci (landmark Ed), 20, 1116-1143. 
West, J. B. (2011). Respiratory physiology: the essentials (9th ed.). Lippincott Williams \& Wilikins.

Wishart, D. S., Feunang, Y. D., Marcu, A., Guo, A. C., Liang, K., Vazquez-Fresno, R., Sajed, T., Johnson, D., Li, C., Karu, N., Sayeeda, Z., Lo, E., Assempour, N., Berjanskii, M., Singhal, S., Arndt, D., Liang, Y., Badran, H., Grant, J., ... Scalbert, A. (2018). HMDB 4.0: The human metabolome database for 2018. Nucleic Acids Research, 46, D608-d617.
Yuan, M., Breitkopf, S. B., Yang, X., \& Asara, J. M. (2012). A positive/negative ion-switching, targeted mass spectrometry-based metabolomics platform for bodily fluids, cells, and fresh and fixed tissue. Nature Protocols, 7, 872-881.

Publisher's Note Springer Nature remains neutral with regard to jurisdictional claims in published maps and institutional affiliations. 